\title{
ADP-ribosylation of Drosophila indirect-flight-muscle actin and arthrin by Clostridium botulinum C2 toxin and Clostridium perfringens iota toxin
}

\author{
Ingo JUST, ${ }^{*}$ Emma S. HENNESSEY, $†$ Douglas R. DRUMMOND, $†$ Klaus AKTORIES ${ }^{\star} \ddagger$ and John C. SPARROW $\dagger$ \\ *Institut für Pharmakologie und Toxikologie der Universität des Saarlandes, D-Homburg, Germany, \\ and †Department of Biology, University of York, Heslington, York Y01 5DD, U.K.
}

Purified Drosophila indirect-flight-muscle actin and arthrin, an actin-ubiquitin conjugate, were ADP-ribosylated by Clostridium botulinum $\mathrm{C} 2$ toxin and Clostridium perfringens iota toxin. Phalloidin treatment inhibited the ADP-riboyslation of Drosophila actin and arthrin. Like actin, the ADP-ribosearthrin linkage was sensitive towards hydroxylamine treatment, indicating arginine as the amino acid acceptor. Actin translated in vitro from the indirect-flight-muscle-specific gene $A c t 88 F$ was ADP-ribosylated by $C$. botulinum $\mathrm{C} 2$ toxin and $C$. perfringens iota toxin. Actin from the R177Q mutant of Act88F translated in vivo was not ADP-ribosylated confirming Arg-177 as the ADPribose acceptor. Mutant L176M actin was modified by both toxins, indicating that amino acid 176 of actin does not define the substrate specificity of $C$. botulinum $\mathrm{C} 2$ toxin. Whereas the gene products of various C-terminal mutants of Act $88 F$ translated in vitro (E334K, V339I, E364K, G368E, R372H) were substrates for ADP-ribosylation by $C$. botulinum $\mathrm{C} 2$ toxin and by $C$. perfringens iota toxin, neither toxin modified the $\mathrm{N}$-terminal O-12 deletion mutant.

\section{INTRODUCTION}

Actin is the eukaryotic substrate for several clostridial ADPribosylating toxins (Aktories and Wegner, 1989; Aktories and Just, 1990; Aktories et al., 1992). It has been shown using protein chemistry that actin is ADP-ribosylated at Arg-177 (Vandekerckhove et al., 1987, 1988), a modification that inhibits actin polymerization (Aktories et al., 1986; Schering et al., 1988) and blocks G-actin ATPase activity (Geipel et al. 1989, 1990). ADP-ribosylated actin interacts with the fast growing barbed ends of actin filaments in a capping-protein-like manner to inhibit polymerization of non-modified actin (Weigt et al., 1989; Wegner and Aktories, 1988). Furthermore, recent studies indicate that the toxins ADP-ribosylate actin in complexes with gelsolin thereby altering the nucleation activity of the gelsolin-actin complex (Wille et al., 1992).

The family of actin-ADP-ribosylating toxins comprises Clostridium botulinum C2 toxin (Aktories et al., 1986), Clostridium perfringens iota toxin (Schering et al., 1988; Simpson et al., 1987), Clostridium spiroforme toxin (Popoff and Boquet, 1988; Simpson et al., 1989) and an ADP-ribosyltransferase produced by Clostridium difficile (Popoff et al., 1988). All these toxins are binary in structure and consist of a binding component and a non-linked enzyme component (Aktories and Just, 1990). Clostridial toxins can be divided into two subclasses. The iotalike toxins ( $C$. perfringens iota toxin, $C$. spiroforme toxin and $C$. difficile ADP-ribosyltransferase) are immunologically related and their binding components can replace each other. In contrast, $C$. botulinum $\mathrm{C} 2$ toxin exhibits no immunological cross-reactivity with the iota-like toxins and its binding component is not able to transfer the enzyme component of iota-like toxins into cells (Simpson et al., 1987, 1989).

Furthermore, $C$. botulinum $\mathrm{C} 2$ toxin and $C$. perfringens iota toxin differ in their actin substrate specificity. Whereas iota toxin ADP-ribosylates all actin isoforms studied so far (Schering et al., 1988; Mauss et al., 1990), C2 toxin is not able to use vertebrate skeletal, cardiac or smooth-muscle $\alpha$-actin as substrates (Aktories et al., 1986; Mauss et al., 1990). Here we used Drosophila indirect-flight-muscle actin as a probe for muscle actin to investigate further the substrate specificity of both toxins. We show that Drosophila flight-muscle actin and arthrin (Ball et al., 1987), a ubiquitin-modified actin, both serve as substrates for ADP-ribosylation by clostridial toxins. Furthermore, we report on the toxin-catalysed ADP-ribosylation of various actin mutants translated in vitro in reticulocyte lysate.

\section{MATERIALS AND METHODS}

\section{Materials}

C. botulinum $\mathrm{C} 2$ toxin (Ohishi et al., 1980) and C. perfringens iota toxin (Stiles and Wilkens, 1986) were purified essentially. as described. Phalloidin was a gift from Dr. Schneider (Madaus, Köln, Germany). Nucleotides were from Boehringer (Mannheim, Germany) and $\left[{ }^{32} \mathrm{P}\right] \mathrm{NAD},\left[{ }^{35} \mathrm{~S}\right]$ methionine and $\left[{ }^{3} \mathrm{H}\right]$ leucine from NEN (Bad Homburg, Germany). Reticulocyte lysate was from Amersham (Bucks., U.K.).

\section{Purification of Drosophila indirect-filight-muscle-specific actin}

Indirect-flight-muscle myofibrils were prepared from homogenates of whole Drosophila using procedures slightly modified from those of Saide et al. (1989). Actin was prepared from these myofibrils using a standard protocol for extraction of insect actin (Bullard et al., 1973), with a heating step (Szilagyi and $\mathrm{Lu}, 1982)$ included during extraction of actin from acetone powder with ACEX buffer (10 mM Tris/HCl, $\mathrm{pH} 8.0,0.5 \mathrm{mM}$ ATP, $0.2 \mathrm{mM} \mathrm{CaCl}, 1.0 \mathrm{mM}$ dithiothreitol).

\section{Preparation of Drosophila actin mutants}

The C-terminal mutants (E334K, V339I, E364K, G368E and $\mathrm{R} 372 \mathrm{H}$ ) were made by in vitro mutagenesis of the cloned Act88F gene (Drummond et al., 1991a). The mutants L176M, R177Q and $\Delta \mathrm{O}-12$ were constructed by oligonucleotide-directed mutagenesis as described in Drummond et al. (1992). Transcription and translation of wild-type and mutant actins was carried out in vitro as described by Drummond et al. (1991a). 


\section{ADP-riboyslation assay}

ADP-ribosylation of actin and arthrin purified from Drosophila indirect flight muscle was carried out essentially as described (Aktories et al., 1986; Schering et al., 1988; Just et al., 1990) in a medium containing $2 \mathrm{mM} \mathrm{MgCl}_{2}, 1 \mathrm{mM}$ EDTA, $1 \mathrm{mM}$ dithiothreitol, $1 \mu \mathrm{M}$ NAD, $100 \mu \mathrm{M}$ phenylmethanesulphonyl fluoride, $20 \mu \mathrm{g} / \mathrm{ml}$ leupeptin, $25 \mathrm{mM}$ triethanolamine $/ \mathrm{HCl}$ $(\mathrm{pH} 7.5)$ and $1-5 \mu \mathrm{g}$ of actin and arthrin with $100 \mathrm{ng}$ of iota toxin or $100 \mathrm{ng}$ of $\mathrm{C} 2$ toxin in a total volume of $100 \mu \mathrm{l}$ for $15 \mathrm{~min}$ at $37^{\circ} \mathrm{C}$. ADP-ribosylation of in vitro-translated actin was performed in the same buffer with $2 \mu \mathrm{l}$ of reticulocyte lysate (approx. $10 \mathrm{pg}$ of actin) in a total volume of $10 \mu \mathrm{l}$.

\section{Non-denaturing gel eloctrophoresis}

Non-denaturing gel electrophoresis was performed as described by Safer (1989) in a Bio-Rad mini-Protean II gel system. Samples were prepared as described by Drummond et al. (1991b) by adding $2 \mu \mathrm{l}$ of sample buffer to $10 \mu \mathrm{l}$ of the ADP-ribosylation reaction mixture. After centrifugation for $15 \mathrm{~min}$ at $13000 \mathrm{~g}, 5 \mu \mathrm{l}$ of the supernatant was loaded on to the gel.

\section{Two-dimensional gel electrophoreses}

Two-dimensional gel electrophoresis was performed in Bio-Rad mini-Protean II two-dimensional cells by the method of O'Farrell (1975).

\section{Chemical stability of the ADP-riboso-proteln bond}

The chemical stability of the ADP-ribose bond was studied as described previously (Tsai et al., 1984; Mayer et al., 1988). After the $\left.{ }^{32} \mathrm{P}\right] \mathrm{ADP}$-ribosylation of actin and arthrin, the reaction was terminated by the addition of $100 \mathrm{mM}$ Hepes (pH 7.5) and $2 \%$ SDS. Thereafter, the ADP-ribosylated proteins were treated with $0.5 \mathrm{M} \mathrm{NaCl}$ or $0.5 \mathrm{M}$ hydroxylamine (pH 7.5) for $2 \mathrm{~h}$ at $37^{\circ} \mathrm{C}$ or with $1 \mathrm{mM} \mathrm{HgCl}$ for $30 \mathrm{~min}$ at $37^{\circ} \mathrm{C}$. The trichloroacetic acidprecipitated proteins were dissolved in sample buffer and used for SDS/PAGE (Laemmli, 1970).

\section{RESULTS}

First we studied the ADP-ribosylation of purified indirect-flightmuscle actin by $C$. botulinum $\mathrm{C} 2$ toxin and $C$. perfringens iota toxin. Figure 1 shows that both toxins labelled a protein with $M_{\mathrm{r}}$ of about 42000 in the presence of $\left.{ }^{32} \mathrm{P}\right] \mathrm{NAD}$, indicating that Drosophila indirect-muscle actin is a substrate for $\mathrm{C} 2$ toxin and iota toxin. Surprisingly, a second radiolabelled band with an apparent $M_{\mathrm{r}}$ of about 55000 was observed. This band is most likely caused by ADP-ribosylation of arthrin, a 1:1 covalent complex of actin and ubiquitin found only in the indirect flight muscle of certain types of insects, e.g. Drosophila and Lethocerus (Bullard et al., 1985; Ball et al., 1987). The actin preparation from Drosophila muscle contains more than one actin isoform (results not shown), although the predominant one is the flightmuscle-specific actin. From this, we cannot exclude the possibility that ADP-ribosylation of actin is only of non-flight-muscle actins. However, arthrin is specific for the indirect flight muscle (Bullard et al., 1985; Ball et al., 1987). The observation that arthrin is ADP-ribosylated indicates that the indirect-flightmuscle-specific actin is a substrate for $\mathrm{C} 2$ toxin. This is confirmed by the ADP-ribosylation of actin expressed in vitro from indirectflight-muscle-specific $A c t 88 F$ gene (see below). (a) (b)

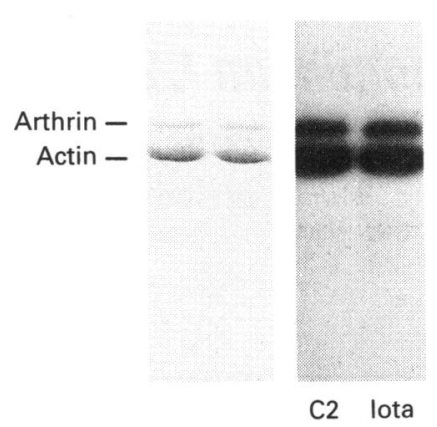

Figure 1 ADP-ribosylation of purfifed Drosophila filght-muscle actin/ arthrin

About $5 \mu \mathrm{g}$ of actin and $1 \mu \mathrm{g}$ of arthrin were ADP-ribosylated in the presence of [ $\left.{ }^{32} \mathrm{P}\right] \mathrm{NAD}$ by C. botulinum $\mathrm{C} 2$ toxin or $C$. perfringens iota toxin as described in the Materials and methods section. The samples were analysed by SDS/PAGE (a) and subsequent autoradiography (b).

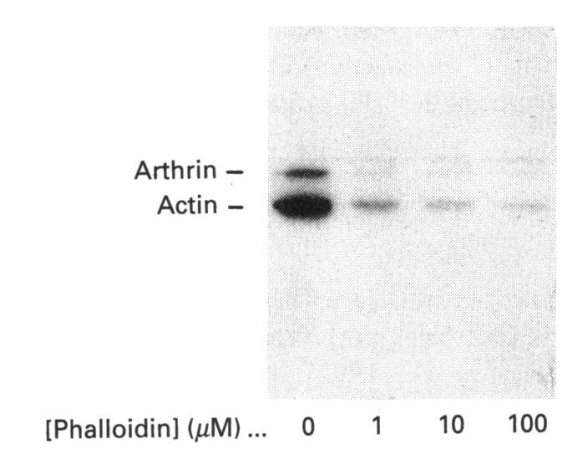

Figure 2 Inhibition of actin/arthrin ADP-ribosylation by phalloidin

Actin/arthrin $(50 / 10 \mu \mathrm{g} / \mathrm{ml})$ was preincubated with phalloidin at the indicated concentrations for $15 \mathrm{~min}$ at $37^{\circ} \mathrm{C}$. Thereafter, ADP-ribosylation was performed by $C$. perfringens iota toxin in the presence of $\left.{ }^{32} \mathrm{P}\right] N A D$ for $15 \mathrm{~min}$ at $37^{\circ} \mathrm{C}$. Autoradiographs of the SDS/ polyacrylamide gels are shown.

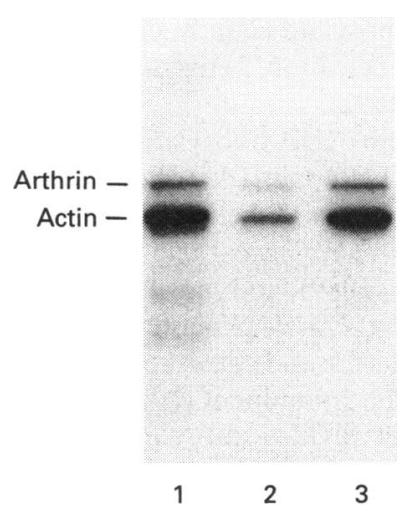

Figure 3 Chemical stability of the ADP-ribose-actin/arthrin bond

[22P]ADP-ribosylated actin/arthrin was denaturated by SDS and treated with $0.5 \mathrm{M} \mathrm{NaCl}$ (control; lane 1), $0.5 \mathrm{M}$ hydroxylamine (pH 7.5) for $2 \mathrm{~h}$ at $37^{\circ} \mathrm{C}$ (lane 2) and $1 \mathrm{mM} \mathrm{HgCl}$ for $30 \mathrm{~min}$ at $37^{\circ} \mathrm{C}$ (lane 3). The proteins were analysed by SDS/PAGE and subsequent autoradiography (shown).

Pretreatment of Drosophila actin with phalloidin, which decreases the critical concentration of actin polymerization (Cooper, 1987), inhibited the ADP-ribosylation by both toxins. Figure 2 shows that increasing concentrations of phalloidin decreased the labelling of Drosophila actin and of arthrin.

Next, we studied the chemical stability of the ADP-ribose-actin linkage towards neutral hydroxylamine. As shown in Figure 3, 


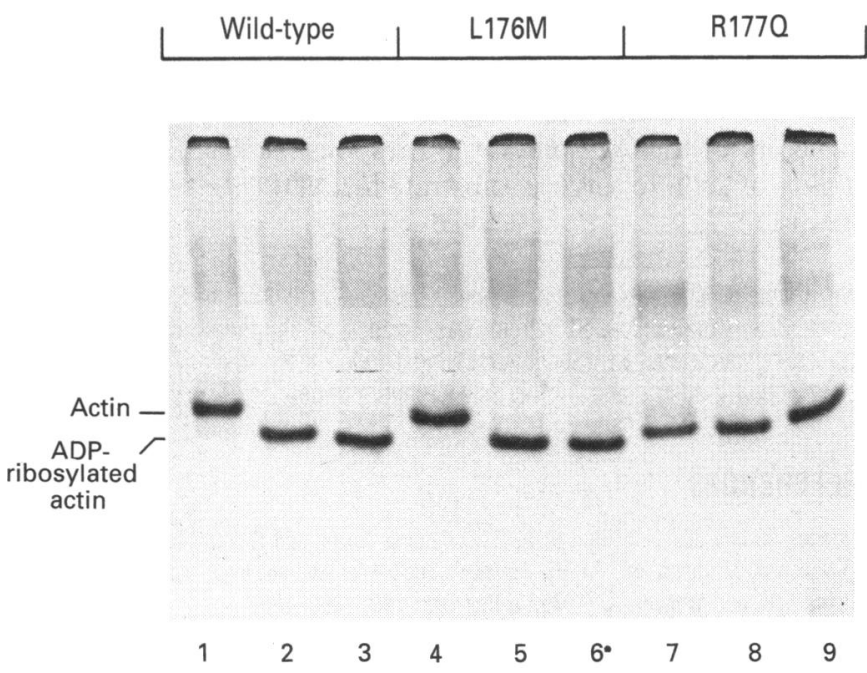

Figure 4 ADP-ribosylation and separation of ${ }^{35}$ S]methionine-labelled in vitro-translated wild-type and mutant (L176M and R177Q) Act88F actin

The wild-type (lanes 1-3) and mutant [L176M (lanes 4-6) and R177Q (lanes 7-9)] actins were ADP-ribosylated by $C$. botulinum $C 2$ toxin (lanes 3,6 and 9 ) and $C$. perfringens iota toxin (lanes 2, 5 and 8 ) and subsequently separated by non-denaturing gel electrophoresis. The autoradiograph is shown. Lanes 1,4 and 7 show controls.

treatment of ADP-ribosylated actin and arthrin for $2 \mathrm{~h}$ with $0.5 \mathrm{M}$ hydroxylamine largely reduced the labelling, indicating that the ADP-ribose moiety is bound to arginine (Hsia et al., 1985; Aktories et al., 1988). $\mathrm{HgCl}_{2}$ treatment, which cleaves the ADP-ribose-cysteine bond (Mayer et al., 1988), had no effect.

By using Drosophila indirect-flight-muscle actin mutants, we attempted to test two hypotheses. First, it has been claimed from the results of chemical analysis that Arg-177 is the residue that is ADP-ribosylated (Vandekerckhove et al., 1987, 1988). Second, it has been suggested that the substrate specificity of $C$. botulinum $\mathrm{C} 2$ toxin might be defined by the amino acid that precedes Arg177: it is leucine in all $\beta / \gamma$ non-muscle actins and methionine in $\alpha$-actin isoforms. For this purpose, the R177Q and L176M mutants of the $A c t 88 F$ flight-muscle gene were constructed and expressed in vitro in rabbit reticulocyte lysate. Subsequently, the mutant proteins were [ $\left.{ }^{32} \mathrm{P}\right] \mathrm{ADP}$-ribosylated by the toxins. However, analysis of the labelled proteins by SDS/PAGE was hampered by the fact that the reticulocyte lysate contained small amounts of endogenous rabbit cytoplasmic actin which was also substrate for ADP-ribosylation (results not shown). Therefore the newly expressed actin was labelled with $\left[{ }^{35}\right.$ S $]$ methionine, then ADP-ribosylated in the presence of unlabelled NAD and the proteins were analysed by non-denaturing gel electrophoresis. Figure 4 shows that the ADP-ribosylation changed the migration behaviour of $\left.{ }^{35} S\right]$ methionine-labelled wild-type actin which has leucine in position 176. A similar change in migration was observed for the L176M mutant actin after ADP-ribosylation by $C$. botulinum $\mathrm{C} 2$ toxin and $C$. perfringens iota toxin, indicating that the mutant actin was a substrate for both toxins. In contrast, no change in the migration of actin was observed when the R177Q mutant was ADP-ribosylated, indicating that neither $C$. botulinum $\mathrm{C} 2$ toxin nor $C$. perfringens iota toxin was able to ADP-ribosylate actin with glutamine at position 177 . R177Q actin has a different mobility on the gels because of its different charge. These results were corroborated by using twodimensional analysis of the ADP-ribosylated actin. Figure 5 shows that the ADP-ribosylation of Drosophila wild-type actin did not cause major changes in the $M_{\mathrm{r}}$ of actin, but the pI was shifted to more acidic values. This also held true for the $\mathrm{L} 176 \mathrm{M}$ mutant but not for the R177Q mutant of the Act88F gene product. We studied the ADP-ribosylation of the E334K, V339I, E364K, G368E and R372H actin mutants which have been shown to be antimorphic in vivo (Drummond et al., 1991a). All of them were substrates for $C$. botulinum $\mathrm{C} 2$ and $C$. perfringens iota toxin (results not shown). A mutant actin that lacked the initial 12 amino acids at the $\mathrm{N}$-terminus migrated much more slowly on the non-denaturing gel, which is most probably due to loss of acidic amino acids. However, neither C. botulinum C2 toxin nor $C$. perfringens iota toxin induced changes in the migration compared with controls, indicating that the 0-12 deletion mutant was not modified by ADP-ribosylation (results not shown).

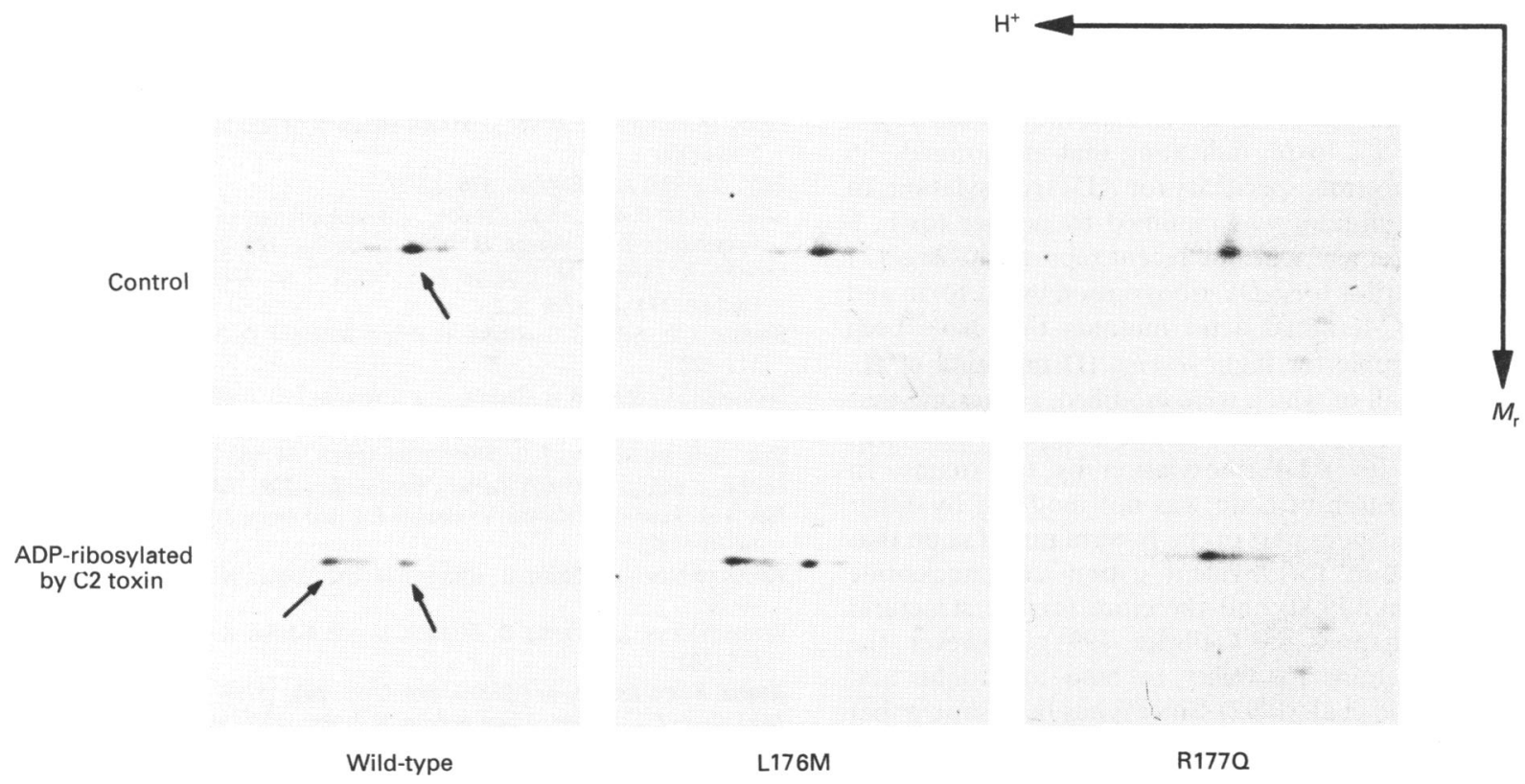

Figure 5 Autoradiographs of two-dimensional gels of [S]methionine-labelled wild-type and mutant Act88F actin ADP-ribosylated by C. botullnum C2 toxin 


\section{DISCUSSION}

Here we report that Drosophila melanogaster indirect-flightmuscle actin was ADP-ribosylated by $C$. botulinum $\mathrm{C} 2$ toxin and $C$. perfringens iota toxin. This is the first demonstration of ADPribosylation by $\mathrm{C} 2$ toxin of a striated muscle actin isoform, although the amino acid sequence of $A c t 88 F$ actin is closer to that of vertebrate cytoplasmic than vertebrate muscle actins. Furthermore, arthrin, a ubiquitinated actin derivative (Bullard et al., 1985; Ball et al., 1987), was also modified by the toxins. Arthrin is known to share most properties with actin; for example, it co-polymerizes with actin and binds myosin (Bullard et al., 1985; Ball et al., 1987) and also DNAase I and profilin (E. S. Hennessey, unpublished work). So far the precise site of the covalent binding of ubiquitin to actin is not known. The finding that arthrin is a substrate for ADP-ribosylation suggests that Arg-177, the acceptor for ADP-ribosylation, and the acceptor site for ubiquitin are located at completely different sites of the actin molecule. This notion is further supported by the findings that unmodified arthrin is still able to polymerize whereas ADP-ribosylated actin, e.g. $\beta / \gamma$-actin (Aktories et al., 1986) or skeletal-muscle actin (Schering et al., 1988), cannot polymerize.

Our findings that the ADP-ribosylation of actin and arthrin was inhibited by phalloidin, which induces actin polymerization, indicate that the polymerized forms of both proteins are no longer substrates for ADP-ribosylation. This observation agrees with former reports that mammalian G-actin but not F-actin is a substrate for ADP-ribosylation (Aktories et al., 1986; Schering et al., 1988). Furthermore, the ADP-ribose-protein bonds of actin and of arthrin were sensitive towards hydroxylamine, a finding that indicates that arthrin is also ADP-ribosylated at an arginine, probably Arg-177 of actin (Aktories et al., 1988; Vandekerckhove et al., 1987, 1988).

C. botulinum $\mathrm{C} 2$ toxin is characterized by a remarkable substrate specificity, because no $\alpha$-actin isoforms (skeletal, cardiac and smooth muscle) are modified by the toxin (Mauss et al., 1990). In contrast, $C$. perfringens iota toxin ADP-ribosylates all actin isoforms studied so far. It has been suggested that the amino acid that precedes Arg-177, namely leucine in non-muscle actin and methionine in $\alpha$-actin isoforms, defines the substrate specificity of C2 toxin (Vandekerckhove et al., 1988). Here we show that the Drosophila Act $88 F$ gene actin, a muscle actin isoform that possesses leucine in position 176, was ADPribosylated by $\mathrm{C} 2$ toxin. However, the L176M mutant of $A c t 88 F$ was still modified by $C 2$ toxin, indicating that amino acid 176 does not define the substrate specificity for ADP-ribosylation. In contrast, the R177Q mutant was modified by neither toxin, a finding that is in agreement with the recent report that Arg-177 is the amino acid acceptor for ADP-ribosylation by $\mathrm{C} 2$ toxin and iota toxin. Several C-terminal actin mutants that have been shown to be antimorphic for flight in vivo (Drummond et al., 1991a) were studied, all of which were modified, suggesting that the amino acids Glu-334, Val-339, Glu-364, Gly-368 and Arg372 are not crucial for ADP-ribosylation by the toxins. In contrast, the $0-12$ deletion of actin was not modified by either toxin. This deletion includes part of the $\mathrm{N}$-terminus of actin that is apparently important for bivalent cation and nucleotide binding (Kabsch et al., 1990) and therefore for the structural integrity of actin (Johannes and Gallwitz, 1991). However, the $\Delta$ 0-12 mutant still retains its ability to bind to profilin and DNAase I (Drummond et al., 1992). Since it has been shown that the ADP-ribosylation of actin depends on the native structure of actin (e.g. treatment of actin with EGTA inhibits ADPribosylation), it is not clear whether the deletion of the mobile $\mathrm{N}$ terminus of actin itself or gross changes in the overall actin structure of the 0-12 mutant are responsible for the loss of the ability of actin to serve as substrate for ADP-ribosylation by the toxins.

We gratefully acknowledge the technical assistance of Annette Stüben and Anne Lawn. The work described herein was financially supported by the Deutsche Forschungsgemeinschaft (Sonderforschungsbereich 246; B9) by the Fonds der Chemischen Industrie and by the Science and Engineering Research Council.

\section{REFERENCES}

Aktories, K. and Just, I. (1990) in ADP-ribosylating Toxins and G-proteins (Moss, J. and Vaughan, M., eds.), pp. 79-95, American Society for Microbiology, Washington, D.C. Aktories, K. and Wegner, A. (1989) J. Cell Biol. 109, 1385-1387

Aktories, K., Bärmann, M., Ohishi, I., Tsuyama, S., Jakobs, K. H. and Habermann, E. (1986) Nature (London) 322, 390-392

Aktories, K., Just, I. and Rosenthal, W. (1988) Biochem. Biophys. Res. Commun. 156, 361-367

Aktories, K., Wille, M. and Just, I. (1992) Curr. Top. Microbiol. Immunol. 175, 97-113 Ball, E., Karlik, C. C., Beall, C. J., Saville, D. L., Sparrow, J. C., Bullard, B. and Fryberg, E. A. (1987) Cell 51, 221-228

Bullard, B., Dabrowska, R. and Winkelman, L. (1973) Biochem. J. 135, 277-286

Bullard, B., Bell, J. and Craig, R. (1985) J. Mol. Biol. 182, 443-454

Cooper, J. A. (1987) J. Cell Biol. 105, 1473-1478

Drummond, D. R., Hennessey, E. S. and Sparrow, J. C. (1991a) Mol. Gen. Genet. 226, $70-80$

Drummond, D. R., Hennessey, E. S. and Sparrow, J. C. (1991b) Biochem. J. 274, 301-303 Drummond, D., Hennessey, E. and Sparrow, J. (1992) Eur. J. Biochem. 209, 171-179 Geipel, U., Just, I., Schering, B., Haas, D. and Aktories, K. (1989) Eur. J. Biochem. 179, 229-232

Geipel, U., Just, I. and Aktories, K. (1990) Biochem. J. 266, 335-339

Hsia, J. A., Tsai, S-C., Adamik, R., Yost, D. A., Hewlett, E. L. and Moss, J. (1985) J. Biol. Chem. 260, 16187-16191

Johannes, F.-J. and Gallwitz, D. (1991) EMBO J. 10, 3951-3958

Just, I., Geipel, U., Wegner, A. and Aktories, K. (1990) Eur. J. Biochem. 192, 723-727

Kabsch, W., Mannherz, H. G., Suck, D., Pai, E. F. and Holmes, K. C. (1990) Nature (London) 347, 37-44

Laemmli, U. K. (1970) Nature (London) 227, 680-685

- Mauss, S., Chaponnier, C., Just, I., Aktories, K. and Gabbiani, G. (1990) Eur. J. Biochem. 194, 237-241

Mayer, T., Koch, R., Fanick, W. and Hilz, H. (1988) Biol. Chem. Hoppe-Seyler 369, $579-583$

O'Farrell, P. H. (1975) J. Biol. Chem. 250, 4007-4021

Ohishi, I., Iwasaki, M. and Sakaguchi, G. (1980) Infect. Immun. 30, 668-673

Popoff, M. R. and Boquet, P. (1988) Biochem. Biophys. Res. Commun. 152, 1361

Popoff, M. R., Rubin, E. J., Gill, D. M. and Boquet, P. (1988) Infect. Immun. 56, 2299-2306

Safer, D. (1989) Anal. Biochem. 178, 32-37

Saide, J., Chin-Bow, S., Hogan-Sheldon, J., Busquets-Turner, L., Vigoreaux, J., Valgeirsdottir, K. and Pardue, M. (1989) J. Cell Biol. 109, 2157-2167

Schering, B., Bärmann, M., Chhatwal, G. S., Geipel, U. and Aktories, K. (1988) Eur. J. Biochem. 171, 225-229

Simpson, L. L., Stiles, B. G., Zapeda, H. H. and Wilkins, T. D. (1987) Infect. Immun. 55, 118-122

Simpson, L. L., Stiles, B. G., Zepeda, H. and Wilkins, T. D. (1989) Infect. Immun. 57, 255-261

Stiles, B. G. and Wilkens, T. D. (1986) Infect. Immun. 54, 683-688

Szilagyi, L. and Lu, R. (1982) Biochem. Biophys. Acta 709, 204-211

Tsai, S. C., Adamik, R., Kanaho, Y., Hewlett, E. L. and Moss, J. (1984) J. Biol. Chem. 259, 15320-15323

Vandekerckhove, J., Schering, B., Bärmann, M. and Aktories, K. (1987) FEBS Lett. 225, 48-52

Vandekerckhove, J., Schering, B., Bärmann, M. and Aktories, K. (1988) J. Biol. Chem. 263, 696-700

Wegner, A. and Aktories, K. (1988) J. Biol. Chem. 263, 13739-13742

Weigt, C., Just, I., Wegner, A. and Aktories, K. (1989) FEBS Lett. 246, 181-184

Wille, M., Just, I., Wegner, A. and Aktories, K. (1992) J. Biol. Chem. 267, 50-55 